\title{
Multi-Strip and Multi-Point Boundary Conditions for Fractional Langevin Equation
}

\author{
Ahmed Salem * and Balqees Alghamdi \\ Department of Mathematics, Faculty of Science, King Abdulaziz University, P.O.Box 80203, Jeddah 21589, \\ Saudi Arabia; aisharif@ju.edu.sa \\ * Correspondence: asaalshreef@kau.edu.sa
}

Received: 9 March 2020; Accepted: 24 April 2020; Published: 28 April 2020

\begin{abstract}
In the present paper, we discuss a new boundary value problem for the nonlinear Langevin equation involving two distinct fractional derivative orders with multi-point and multi-nonlocal integral conditions. The fixed point theorems for Schauder and Krasnoselskii-Zabreiko are applied to study the existence results. The uniqueness of the solution is given by implementing the Banach fixed point theorem. Some examples showing our basic results are provided.
\end{abstract}

Keywords: fixed point theorem; fractional Langevin equations; existence and uniqueness solution

MSC: 26A33; 34A08; 34A12; 34B15

\section{Introduction}

The Langevin equation was discovered by Langevin a century ago to render an accurate description of the evolution of physical phenomena in fluctuating environments. This equation can be considered as a special form of the generalized Langevin equation [1], which has turned into a modern research project theme.

Fractional calculus has attracted many authors and researchers in many different scientific disciplines. Many of the recent advances in fractional calculus were motivated by the modern applications of fractional integro-differential equations in various fields, in particular physics. One of the main reasons for its popularity in modeling various transport properties in complex heterogeneous and disordered media is that it provides a natural setting for describing processes with memory and is fractal or multi-fractal in nature [2]. For systems in complex media, the ordinary Langevin equation does not provide the correct description of the dynamics. Various generalizations of Langevin equations have been proposed to describe dynamical processes in a fractal medium. One such generalization is the Langevin equation with two fractional orders, which incorporates the fractal and memory properties with a dissipative memory kernel into the Langevin equation. This possible extension requires the replacement of the ordinary derivative by a fractional derivative in the Langevin equation to give the fractional Langevin equation [3-5]. Various versions of fractional Langevin-type equations have been proposed to model anomalous diffusion [6,7], and both deterministic and stochastic fractional equations are used to describe non-Debye dielectric relaxation phenomena.

Anomalous diffusion has been found in various physical and biological systems. The mean squared displacement of the particle shows a power law dependence on time $\left\langle x^{2}(t)\right\rangle \sim t^{\alpha}$, becoming subdiffusion in the case $0<\alpha<1$, superdiffusion for $\alpha>1$, and normal classical diffusion for $\alpha=1$ [8-10]. Several stochastic approaches to anomalous diffusion exist. In most cases, such behavior is considered to be connected with the self-similar properties of the diffusion medium. As this took place, the generalized Langevin equation came to fame following Kubos' work and the related fractional Brownian motion, originally introduced by Kolmogorov [11] and popularized by Mandelbrot [12]. 
It is remarkable to note that Mainardi and Pironi [13] introduced a fractional Langevin equation as a particular case of a generalized Langevin equation and for the first time represented the velocity and displacement correlation functions in terms of the Mittag-Leffler functions.

Due to the extremely useful role of the fractional Langevin equation in applied mathematics, physics, engineering, and several branches of science, it has acquired many scientific contributions in the field of finding exact solutions $[3,14,15]$, approximate solutions through numerical analysis methods [16-18], and studying the existence and uniqueness of the solution (see [19-25] and the references given therein). Studying differential equations with integral boundary conditions designates an extremely useful and interesting class of boundary value problems. Several of the problems are in chemical engineering, population dynamics, heat conduction, thermoelasticity, underground water flow, and plasma physics [26]. Furthermore, there are many published contributions concerned with the fractional boundary value problem with the integral boundary conditions (see [27-29] and the references given therein). Multi-point boundary value problems for differential equations become apparent naturally in scientific applications. For an illustration, given a dynamical system with $m$ degrees of freedom, there may be available exactly $m$ cases spotted at $m$ distinct times. A mathematical depiction of such problems is in an $m$-point boundary value problem. Multi-point problems for differential equations are a special class of interface problems, and hence solvable with various techniques. Studying fractional differential equations with multi-pint boundary condition has been drawn the attention of many contributors (see [30-33] and the references given therein).

Motivated by the significance of the integral boundary conditions and fractional Langevin equations in different branches of science and engineering, this paper is interested in studying the nonlinear fractional Langevin equation:

$$
{ }^{c} D^{p}\left({ }^{c} D^{q}+\mu\right) x(t)=f\left(t, x(t),{ }^{c} D^{r} x(t)\right), \quad t \in[0,1]
$$

with the new auxiliary multi-integral and multi-point boundary conditions:

$$
x(0)=0, \quad D^{q} x(0)=0, \quad x(1)=\sum_{i=1}^{n} \alpha_{i} x\left(\eta_{i}\right)+\sum_{i=1}^{n} \beta_{i} \int_{0}^{\eta_{i}} x(s) d s
$$

where ${ }^{c} D^{p},{ }^{c} D^{q}$, and ${ }^{c} D^{r}$ are the Liouville-Caputo fractional derivative of orders $p \in(1,2], q \in(0,1]$, and $0<r \leq q, \mu \in \mathbb{R}$ is the dissipative parameter, $\mu, \alpha_{i}, \beta_{i} \in \mathbb{R}, \eta_{i} \in(0,1), i=1,2, \cdots, n$ such that $n \in \mathbb{N}$ with $\omega=\sum_{i=1}^{n} \alpha_{i} \eta_{i}{ }^{q+1} \neq 1$, and the function $f:[0,1] \times \mathbb{R} \times \mathbb{R} \rightarrow \mathbb{R}$ is continuous.

It is worth mentioning that $x(t)$ in Equation (1) is the displacement of the particle in the general interval $t \in[0, a], a>0$ (for simplicity, we apply the transformation $t=t / a$ to make $t$ in the unit interval $[0,1])$. Instead of the ordinary definition of the velocity and acceleration as the first and second derivatives of the displacement, respectively, we render fractional forms ${ }^{c} D^{\alpha} x(t), 0<\alpha \leq 1$ for the velocity and ${ }^{c} D^{\beta} x(t), 1<\beta \leq 2$ for the the acceleration. The product of two fractional derivatives gives the term ${ }^{c} D^{\alpha+\beta}$, which represents the acceleration if $1<\alpha+\beta \leq 2$ and the aberrancy of the curve or the Jerk term [34-36] if $2<\alpha+\beta \leq 3$ instead of defining it as a third time derivative of the displacement. We take here the function $f$ in the general form, which is constituted by the position $x(t)$ and velocity ${ }^{c} D^{r} x(t)$ of the particle at time $t$. This function may contain an external force field, a position-dependent phenomenological fluid friction coefficient, the intensity of the stochastic force, or the zero-mean Gaussian white noise term.

The first and second boundary conditions in Equation (2) indicate that the particle begins its motion from stillness at the origin. The last condition in Equation (2), which seems to be a linear combination of the values of the unknown function at the multi-point and multi-strip, can be interpreted as "the value of of the unknown function at the terminal point proportionate to the summation of values of it at midst nonlocal $m$-points and the areas under its curve from the initial point to the midst points". 
In mathematical analysis, the existence and uniqueness of the solution for differential and integral equations have become major topics. There are many fixed point theorems used to discuss the existence results [37]. One of these theorems due to Krasnoselskii-Zabreiko lacks usage, although it gives more precise sufficient conditions of the existence results. This inspired us to discuss the existence of the solution for our problem by implementing the Krasnoselskii-Zabreiko fixed point theorem. Furthermore, we apply the Schauder fixed point under different assumptions and show its applicability by means of studying a numerical example. In addition, the uniqueness of the solution is investigated by applying the Banach fixed point theorem.

The strategy of the paper is as follows: In the section below, we render some definitions and results that are needed in this paper. The existence and uniqueness are discussed in Section 3. In the last section, we establish some examples to show these results.

Introducing the fractional element provides many possibilities for the generalizations of models described in the previous subsection.

\section{Preliminaries}

Throughout this section, the definitions needed and the notations are given. Let $C[0,1]$ be the class continuous functions on $[a, b]$. Furthermore, let $A C[a, b]$ be the space of functions $f$ that are absolutely continuous on $[a, b]$. For $n \in \mathbb{N}$, we denote by $A C^{n}[a, b]$ the space of real functions $f(t)$, which have continuous derivatives up to order $n-1$ on $[a, b]$ such that $f^{(n-1)}(t) \in A C[a, b]$. In particular, $A C^{1}[a, b]=A C[a, b][38]$.

Definition 1 ([38]). If $x(t) \in C[a, b]$, then, the $R$-Lfractional integral with order $p>0$ exists almost everywhere on $[a, b]$ and can be represented in the form:

$$
I^{p} x(t)=\frac{1}{\Gamma(p)} \int_{a}^{t}(t-s)^{p-1} x(s) d s .
$$

Definition 2 ([38]). If $x(t) \in A C^{n}[a, b]$ and $n \in \mathbb{N}$, the Liouville-Caputo fractional derivative of order $n-1<p \leq n$ exists almost everywhere on $[a, b]$ and can be represented in the form:

$$
{ }^{c} D^{p} x(t)=\frac{1}{\Gamma(n-p)} \int_{a}^{t}(t-s)^{n-p-1} x^{(n)}(s) d s .
$$

Lemma 1 ([38,39]). Let $n \in \mathbb{N}, n-1<q \leq n$, and $x(t) \in C^{n}[0,1]$, then we have:

$$
I^{q c} D^{q} x(t)=x(t)+a_{0}+a_{1} t+\cdots+a_{n-1} t^{n-1}
$$

Lemma 2 ([38,39]). Let $p>0, n \in \mathbb{N}$ such that $n-1<q \leq n$, then:

$$
\begin{array}{ll}
1 & { }^{c} D^{q} I^{p} x(t)=D^{q-p} x(t) \text { if } q>p, \\
2 & { }^{c} D^{q} I^{p} x(t)=I^{p-q} x(t) \text { if } p>q .
\end{array}
$$

Lemma 3. Suppose the function $g: C[0,1] \rightarrow \mathbb{R}$; hence, the unique solution of the linear equation:

$$
{ }^{c} D^{p}\left({ }^{c} D^{q}+\mu\right) x(t)=g(t), \quad t \in[0,1]
$$


with the conditions mentioned in Equation (2), can be taken the form:

$$
\begin{aligned}
x(t) & =\frac{1}{\Gamma(q+p)} \int_{0}^{t}(t-s)^{q+p-1} g(s) d s-\frac{\mu}{\Gamma(q)} \int_{0}^{t}(t-s)^{q-1} x(s) d s \\
& +\frac{t^{q+1}}{(1-\omega)}\left[\frac{\mu}{\Gamma(q)} \int_{0}^{1}(1-s)^{q-1} x(s) d s-\frac{1}{\Gamma(p+q)} \int_{0}^{1}(1-s)^{q+p-1} g(s) d s\right. \\
& +\sum_{i=1}^{n} \frac{\alpha_{i}}{\Gamma(p+q)} \int_{0}^{\eta_{i}}\left(\eta_{i}-s\right)^{q+p-1} g(s) d s-\mu \sum_{i=1}^{n} \frac{\alpha_{i}}{\Gamma(q)} \int_{0}^{\eta_{i}}\left(\eta_{i}-s\right)^{q-1} x(s) d s \\
& \left.+\sum_{i=1}^{n} \beta_{i} \int_{0}^{\eta_{i}} x(s) d s\right]
\end{aligned}
$$

Proof. Applying Lemma 1, we get:

$$
{ }^{c} D^{q} x(t)=I^{p} g(t)-\mu x(t)+a_{0}+a_{1} t
$$

Furthermore, we apply Lemma 1 and use the relation $I^{q} t^{p}=\frac{\Gamma(p+1)}{\Gamma(p+q+1)} t^{p+q}$, and Equation (5) becomes:

$$
\begin{aligned}
x(t) & =\frac{1}{\Gamma(q+p)} \int_{0}^{t}(t-s)^{q+p-1} g(s) d s-\frac{\mu}{\Gamma(q)} \int_{0}^{t}(t-s)^{q-1} x(s) d s \\
& +\frac{t^{q}}{\Gamma(q+1)} a_{0}+\frac{t^{q+1}}{\Gamma(q+2)} a_{1}+a_{2}
\end{aligned}
$$

By using the boundary conditions $x(0)=0$ and $D^{q} x(0)=0$ in Equations (5) and (6), respectively, we find that $a_{0}=0$ and $a_{2}=0$. The boundary equation $x(1)=\sum_{i=1}^{n} \alpha_{i} x\left(\eta_{i}\right)+\sum_{i=1}^{n} \beta_{i} \int_{0}^{\eta_{i}} x(s) d s$ in Equation (6) gives the value of the constant $a_{1}$ as:

$$
\begin{aligned}
a_{1} & =\frac{\Gamma(q+2)}{(1-\omega)}\left[\mu I^{q} x(1)-I^{p+q} g(1)+\sum_{i=1}^{n} \alpha_{i} I^{p+q} g\left(\eta_{i}\right)-\mu \sum_{i=1}^{n} \alpha_{i} I^{q} x\left(\eta_{i}\right)\right. \\
& \left.+\sum_{i=1}^{n} \beta_{i} \int_{0}^{\eta_{i}} x(s) d s\right]
\end{aligned}
$$

Substitute the values $a_{0}, a_{1}$ and $a_{2}$ in Equation (6) to obtain Equation (4). Conversely, inserting Equation (4) in the left side of Equation (3) using Lemma 2 implies the right side. Furthermore, it is not difficult to see that Equation (4) verifies the boundary condition Equation (2). This completes the proof.

\section{Main Results}

Define the space:

$$
X=\left\{x: x \in C[0,1],{ }^{c} D^{r} x \in C[0,1], 0<r \leq 1\right\}
$$

equipped with the norm:

$$
\|x\|_{X}=\|x\|+\left\|{ }^{c} D^{r} x(t)\right\|=\max _{t \in[0,1]}|x(t)|+\max _{t \in[0,1]}\left|{ }^{c} D^{r} x(t)\right| .
$$

It is worth pointing out that $\mathrm{Su}[40]$ proved that $X$ is a Banach space equipped with the former norm.

Assume the following hypotheses that we need to prove the existence and uniqueness results of the problem Equations (1) and (2).

$\left(G_{1}\right) f:[0,1] \times \mathbb{R} \times \mathbb{R} \rightarrow \mathbb{R}$ is a continuous function; 
$\left(G_{2}\right)$ There exists a positive function $\psi \in X$ such that $|f(t, x, y)| \leq \psi(t)+a_{1}|x|^{r_{1}}+a_{2}|y|^{r_{2}}$ where $a_{1}, a_{2} \in \mathbb{R}^{+}$and $0<r_{1}, r_{2} \leq 1 ;$

$\left(G_{3}\right)$ The continuous function $f(t, 0,0)$ does not vanish identically in $[0,1]$;

$\left(G_{4}\right) \lim _{r \rightarrow \infty} \frac{f(t, x(t), y(t))}{x(t)+y(t)}=\kappa(t)$ uniformly in $[0,1]$ where $x, y \in X, r=\|x\|+\|y\|$ and $\kappa:[0,1] \rightarrow \mathbb{R}$ is continuous;

(G) There exists a constant $L>0$ such that:

$$
|f(t, x, y)-f(t, \hat{x}, \hat{y})| \leq L(|x-\hat{x}|+|y-\hat{y}|), \quad t \in[0,1], x, \hat{x}, y, \hat{y} \in \mathbb{R}
$$

For convenience, let:

$$
\begin{aligned}
& \Theta=\Theta_{p, r}+\Theta_{p, 0}, \\
& \mathrm{Y}=\mathrm{Y}_{r}+\mathrm{Y}_{0}
\end{aligned}
$$

where:

$$
\begin{aligned}
& \Theta_{p, r}=\frac{1}{\Gamma(p+q-r+1)}+\frac{\Gamma(q+2)}{|1-\omega| \Gamma(q-r+2) \Gamma(p+q+1)}\left(1+\sum_{i=1}^{n} \alpha_{i} \eta_{i}^{p+q}\right) \\
& \mathrm{Y}_{r}=|\mu| \Theta_{0, r}+\frac{\Gamma(q+2)}{|1-\omega| \Gamma(q-r+2)} \sum_{i=1}^{n} \beta_{i} \eta_{i}
\end{aligned}
$$

We express the operator $T: X \rightarrow X$ as:

$$
\begin{aligned}
(T x)(t) & =\frac{1}{\Gamma(q+p)} \int_{0}^{t}(t-s)^{q+p-1} f\left(s, x(s),{ }^{c} D^{r} x(s)\right) d s-\frac{\mu}{\Gamma(q)} \int_{0}^{t}(t-s)^{q-1} x(s) d s \\
& +\frac{t^{q+1}}{1-\omega}\left[T_{1}(x)+T_{2}(f)\right]
\end{aligned}
$$

and its $r$ th Caputo fractional derivative:

$$
\begin{aligned}
{ }^{c} D^{r}(T x)(t) & =\frac{1}{\Gamma(q+p-r-1)} \int_{0}^{t}(t-s)^{q+p-1} f\left(s, x(s),{ }^{c} D^{r} x(s)\right) d s \\
& -\frac{\mu}{\Gamma(q-r)} \int_{0}^{t}(t-s)^{q-r-1} x(s) d s+\frac{\Gamma(q+2) t^{q+1-r}}{(1-\omega) \Gamma(q-r+2)}\left[T_{1}(x)+T_{2}(f)\right]
\end{aligned}
$$

where:

$$
\begin{aligned}
T_{1}(x) & =\frac{\mu}{\Gamma(q)} \int_{0}^{1}(1-s)^{q-1} x(s) d s-\mu \sum_{i=1}^{n} \frac{\alpha_{i}}{\Gamma(q)} \int_{0}^{\eta_{i}}\left(\eta_{i}-s\right)^{q-1} x(s) d s+\sum_{i=1}^{n} \beta_{i} \int_{0}^{\eta_{i}} x(s) d s \\
T_{2}(f) & =\sum_{i=1}^{n} \frac{\alpha_{i}}{\Gamma(p+q)} \int_{0}^{\eta_{i}}\left(\eta_{i}-s\right)^{q+p-1} f\left(s, x(s),{ }^{c} D^{r} x(s)\right) d s \\
& -\frac{1}{\Gamma(p+q)} \int_{0}^{1}(1-s)^{q+p-1} f\left(s, x(s),{ }^{c} D^{r} x(s)\right) d s .
\end{aligned}
$$

It is easy to see that:

$$
\begin{aligned}
& \left\|T_{1}(x)\right\| \leq \frac{\|x\|}{\Gamma(q+1)}\left(|\mu|+|\mu| \sum_{i=1}^{n}\left|\alpha_{i}\right| \eta_{i}^{q}+\Gamma(q+1) \sum_{i=1}^{n}\left|\beta_{i}\right| \eta_{i}\right) \\
& \left\|T_{2}(f)\right\| \leq \frac{\|f\|}{\Gamma(p+q+1)}\left(1+\sum_{i=1}^{n}\left|\alpha_{i}\right| \eta_{i}^{q+p}\right)
\end{aligned}
$$


Our first result is discussing the existence of the solution for the problem by the Schauder fixed point theorem.

Theorem 1. Assume that $G_{1}$ and $G_{2}$ hold. Then, the boundary value problem Equations (1) and (2) have a solution.

Proof. We express the operator $T: X \rightarrow X$ and let a closed ball $\mathcal{B}_{\xi}=\left\{x \in X:\|x\|_{X} \leq \xi\right\}$ taking:

$$
\xi>\max \left\{4\|\psi\|_{X} \Theta,\left(4 a_{1} \Theta\right)^{\frac{1}{1-r_{1}}},\left(4 a_{2} \Theta\right)^{\frac{1}{1-r_{2}}}, 4 \xi Y\right\}
$$

Then, we claim that $T \mathcal{B}_{\xi} \subset \mathcal{B}_{\xi}$. For $x \in \mathcal{B}_{\xi}$ and by the condition $G_{1}$, we give:

$$
\begin{aligned}
|(T x)(t)| & \leq \frac{1}{\Gamma(q+p)} \int_{0}^{t}(t-s)^{q+p-1}\left|f\left(s, x(s),{ }^{c} D^{r} x(s)\right)\right| d s+\frac{|\mu|}{\Gamma(q)} \int_{0}^{t}(t-s)^{q-1}|x(s)| d s \\
& +\frac{1}{|1-\omega|}\left[\left|T_{1}(x)\right|+\left|T_{2}(f)\right|\right] \\
& \leq\left(\|\psi\|_{X}+a_{1} \xi^{r_{1}}+a_{2} \xi^{r_{2}}\right)\left\{\frac{t^{p+q}}{\Gamma(p+q+1)}+\frac{1}{(1-\omega) \Gamma(p+q+1)}+\frac{\sum_{i=1}^{n}\left|\alpha_{i}\right| \eta_{i}^{p+q}}{(1-\omega) \Gamma(p+q+1)}\right\} \\
& +\|x\|_{X}\left\{\frac{|\mu| t^{q}}{\Gamma(q+1)}+\frac{t^{q+1}|\mu|}{(1-\omega) \Gamma(q+1)}+\frac{t^{q+1}|\mu|}{(1-\omega) \Gamma(q+1)} \sum_{i=1}^{n} \alpha_{i} \eta_{i}^{q}+\frac{t^{q+1}}{(1-\omega)} \sum_{i=1}^{n}\left|\beta_{i}\right|\left|\eta_{i}\right|\right\} \\
& \leq\left(\|\psi\|_{X}+a_{1} \xi^{r_{1}}+a_{2} \xi^{r_{2}}\right)\left\{\frac{1}{\Gamma(p+q+1)}+\frac{1+\sum_{i=1}^{n}\left|\alpha_{i}\right| \eta_{i}^{p+q}}{(1-\omega) \Gamma(p+q+1)}\right\} \\
& +\xi\left\{\frac{|\mu|}{\Gamma(q+1)}+\frac{|\mu|\left(1+\sum_{i=1}^{n}\left|\alpha_{i}\right| \eta_{i}^{q}\right)}{(1-\omega) \Gamma(q+1)}+\frac{\sum_{i=1}^{n}\left|\beta_{i}\right|\left|\eta_{i}\right|}{1-\omega}\right\} \\
& \leq\left(\|\psi\|_{X}+a_{1} \xi^{r_{1}}+a_{2} \xi^{r_{2}}\right) \Theta_{p, 0}+\xi Y_{0}
\end{aligned}
$$

Similarly, we have:

$$
\left|{ }^{c} D^{r}(T x)(t)\right| \leq\left(\|\psi\|_{X}+a_{1} \xi^{r_{1}}+a_{2} \xi^{r_{2}}\right) \Theta_{p, r}+\xi Y_{r}
$$

Consequently,

$$
\begin{aligned}
& \|T x\|_{X}=\max |(T x)(t)|+\max \left|\left({ }^{c} D^{r}(T x)(t)\right)\right| \\
& \leq\left(\|\psi\|_{X}+a_{1} \xi^{r_{1}}+a_{2} \xi^{r_{2}}\right)\left(\Theta_{p, 0}+\Theta_{p, r}\right)+\xi\left(\mathrm{Y}_{0}+\mathrm{Y}_{r}\right) \\
& \leq\left(\|\psi\|_{X}+a_{1} \xi^{r_{1}}+a_{2} \xi^{r_{2}}\right) \Theta+\xi \mathrm{Y} \\
& \leq \frac{\xi}{4}+\frac{\xi}{4}+\frac{\xi}{4}+\frac{\xi}{4}=\xi
\end{aligned}
$$
We set:

Then, the operator $T: X \rightarrow X$ is uniformly bounded. Next, we show that $T$ is equicontinuous.

$$
\mathrm{N}=\max _{t \in[0,1]}\left|f\left(t, x(t),{ }^{c} D^{r} x(t)\right)\right|+1
$$


and for $x \in \mathcal{B}_{\tilde{\xi}}$, let $t_{1}, t_{2} \in[0,1]$, whereas for $t_{1}<t_{2}$, we get:

$$
\begin{aligned}
& \left|(T x)\left(t_{2}\right)-(T x)\left(t_{1}\right)\right| \leq \mid \frac{1}{\Gamma(p+q)} \int_{0}^{t_{2}}\left(t_{2}-s\right)^{q+p-1} f\left(s, x(s),{ }^{c} D^{r} x(s)\right) d s \\
& -\frac{\mu}{\Gamma(q)} \int_{0}^{t_{2}}\left(t_{2}-s\right)^{q-1} x(s) d s-\frac{1}{\Gamma(p+q)} \int_{0}^{t_{1}}\left(t_{1}-s\right)^{q+p-1} f\left(s, x(s), D^{r} x(s)\right) d s \\
& +\frac{\mu}{\Gamma(q)} \int_{0}^{t_{1}}\left(t_{1}-s\right)^{q-1} x(s) d s|+| \frac{\left(t_{2}{ }^{q+1}-t_{1}^{q+1}\right)}{1-\omega}\left(T_{1}(x)+T_{2}(f)\right) \mid \\
& \leq \mid \frac{1}{\Gamma(p+q)} \int_{0}^{t_{1}}\left[\left(t_{2}-s\right)^{q+p-1}-\left(t_{1}-s\right)^{p+q-1}\right] f\left(s, x(s),{ }^{c} D^{r} x(s)\right) d s \\
& +\frac{1}{\Gamma(p+q)} \int_{t_{1}}^{t_{2}}\left(t_{2}-s\right)^{q+p-1} f\left(s, x(s),{ }^{c} D^{r} x(s)\right) d s \mid \\
& +\left|\frac{\mu}{\Gamma(q)} \int_{0}^{t_{1}}\left[\left(t_{1}-s\right)^{q-1}-\left(t_{2}-s\right)^{q-1}\right] x(s) d s+\frac{\mu}{\Gamma(q)} \int_{t_{1}}^{t_{2}}\left(t_{2}-s\right)^{q-1} x(s) d s\right| \\
& +\frac{t_{2}{ }^{q+1}-t_{1}{ }^{q+1}}{|1-\omega|}\left(\left|T_{1}(x)\right|+\left|T_{2}(f)\right|\right) \\
& \leq \frac{\mathrm{N}}{\Gamma(p+q+1)}\left(t_{2}^{p+q}-t_{1}^{p+q}\right)+\frac{2|\mu| \xi}{\Gamma(q+1)}\left(t_{2}-t_{1}\right)^{q}+\frac{\left(t_{2}{ }^{q+1}-t_{1}{ }^{q+1}\right)}{(1-\omega)}\left\{\xi \frac{|\mu|}{\Gamma(q+1)}\right. \\
& \left.+\frac{\mathrm{N}}{\Gamma(p+q+1)}+\mathrm{N} \frac{\sum_{i=1}^{n}\left|\alpha_{i}\right| \eta_{i}{ }^{p+q}}{\Gamma(p+q+1)}+\xi \frac{|\mu| \sum_{i=1}^{n}\left|\alpha_{i}\right| \eta_{i}{ }^{q}}{\Gamma(q+1)}+\xi \sum_{i=1}^{n}\left|\beta_{i}\right|\left|\eta_{i}\right|\right\}
\end{aligned}
$$

Similarly,

$$
\begin{aligned}
& \left|\left({ }^{c} D^{r} T x\right)\left(t_{2}\right)-{ }^{c} D^{r}(T x)\left(t_{1}\right)\right| \leq \frac{\mathrm{N}}{\Gamma(p+q-r+1)}\left(t_{2}^{p+q-r}-t_{1}^{p+q-r}\right)+\frac{2|\mu| \xi}{\Gamma(q-r+1)}\left(t_{2}-t_{1}\right)^{q-r} \\
& +\frac{\Gamma(q+2)\left(t_{2}{ }^{q-r+1}-t_{1}^{q-r+1}\right)}{\Gamma(q-r+2)(1-\omega)}\left\{\xi \frac{|\mu|}{\Gamma(q+1)}+\frac{\mathrm{N}}{\Gamma(p+q+1)}+\mathrm{N} \frac{\sum_{i=1}^{n}\left|\alpha_{i}\right| \eta_{i}{ }^{p+q}}{\Gamma(p+q+1)}\right. \\
& \left.+\xi \frac{|\mu| \sum_{i=1}^{n}\left|\alpha_{i}\right| \eta_{i}^{q}}{\Gamma(q+1)}+\xi \sum_{i=1}^{n}\left|\beta_{i}\right|\left|\eta_{i}\right|\right\}
\end{aligned}
$$

Observe that $\left(t_{2}^{p+q}-t_{1}^{p+q}\right),\left(t_{2}^{q+1}-t_{1}^{q+1}\right),\left(t_{2}-t_{1}\right)^{q},\left(t_{2}^{p+q-r}-t_{1}^{p+q-r}\right),\left(t_{2}^{q-r+1}-t_{1}^{q-r+1}\right)$, and $\left(t_{2}-t_{1}\right)^{q-r}$ approach uniformly zero as $t_{1}$ approaches $t_{2}$. Then, the operator $T$ is equicontinuous, and we get that the operator $T$ is uniformly bounded since $T \mathcal{B}_{\xi} \subset \mathcal{B}_{\xi}$. Therefore, the Arzela-Ascoli theorem leads to that the operator being completely continuous. Hence, the Schauder fixed point theorem ensures the existence of the solution for problem Equations (1) and (2).

The second result is discussing the existence of the solution by using Krasnoselskii-Zabreiko's fixed point theorem:

Lemma 4 ([41]). Let $\mathcal{W}$ be a Banach space. Suppose that $\mathfrak{F}: \mathcal{W} \rightarrow \mathcal{W}$ is a completely continuous mapping and $\mathfrak{G}: \mathcal{W} \rightarrow \mathcal{W}$ is a bounded linear mapping such that 1 is not an eigenvalue of $\mathfrak{G}$ and:

$$
\lim _{\|x\| \rightarrow \infty} \frac{\|\mathfrak{F} x-\mathfrak{G} x\|}{\|x\|}=0
$$

Then, $\mathfrak{F}$ has a fixed point in $\mathcal{W}$.

Theorem 2. Assume that $G_{1}, G_{3}$, and $G_{4}$ hold. Then, the boundary value problem Equations (1) and (2) have a solution if $\|\kappa\| \Theta+\mathrm{Y}<1$ where $\Theta$ and $\mathrm{Y}$ are defined as in Equations (7) and (8), respectively. 
Proof. Let $f\left(t, x(t),{ }^{c} D^{r} x(t)\right)=\kappa(t)\left(x(t)+{ }^{c} D^{r} x(t)\right)$, and consider the linear operator:

$$
\begin{aligned}
(F x)(t) & =\frac{1}{\Gamma(q+p)} \int_{0}^{t}(t-s)^{q+p-1} \kappa(s)\left(x(s)+{ }^{c} D^{r} x(s)\right) d s-\frac{\mu}{\Gamma(q)} \int_{0}^{t}(t-s)^{q-1} x(s) d s \\
& +\frac{t^{q+1}}{1-\omega}\left[T_{1}(x)+T_{2}\left(\kappa\left(x+{ }^{c} D^{r} x\right)\right)\right]
\end{aligned}
$$

and its fractional derivative:

$$
\begin{aligned}
{ }^{c} D^{r}(F x)(t) & =\frac{1}{\Gamma(q+p-r-1)} \int_{0}^{t}(t-s)^{q+p-1} \mathcal{K}(s)\left(x(s)+{ }^{c} D^{r} x(s)\right) d s \\
& -\frac{\mu}{\Gamma(q-r)} \int_{0}^{t}(t-s)^{q-r-1} x(s) d s \\
& +\frac{\Gamma(q+2) t^{q+1-r}}{(1-\omega) \Gamma(q-r+2)}\left[T_{1}(x)+T_{2}\left(\kappa\left(x+{ }^{c} D^{r} x\right)\right)\right] .
\end{aligned}
$$

Now, we show that one is not an eigenvalue of $F x(t)$ and ${ }^{c} D^{r} F x(t)$. Use the proof by contradiction. Suppose that one is an eigenvalue of $F x(t)$, and ${ }^{c} D^{r} F x(t)$ using Equations (11) and (12), we can deduce that:

$$
\begin{aligned}
\|F x\| & \leq\|x\|_{X}\left\{\frac{\|\kappa\|}{\Gamma(p+q+1)}+\frac{|\mu|}{\Gamma(q+1)}+\frac{|\mu|}{\Gamma(q+1)(1-\omega)}+\frac{\|\kappa\|}{\Gamma(p+q+1)(1-\omega)}\right. \\
& \left.+\frac{\|\kappa\| \sum_{i=1}^{n}\left|\alpha_{i}\right| \eta_{i}^{p+q}}{\Gamma(p+q+1)}+\frac{|\mu| \sum_{i=1}^{n} \eta_{i}{ }^{q}}{\Gamma(q+1)(1-\omega)}+\sum_{i=1}^{n}\left|\beta_{i}\right|\left|\eta_{i}\right|\right\}
\end{aligned}
$$

and:

$$
\begin{aligned}
\left\|{ }^{c} D^{r} F x\right\| & \leq\|x\|_{X}\left\{\frac{\|\kappa\|}{\Gamma(p+q-r+1)}+\frac{|\mu|}{\Gamma(q-r+1)}+\frac{|\mu| \Gamma(q+2)}{\Gamma(q-r+1) \Gamma(q+1)(1-\omega)}\right. \\
& +\frac{\|\kappa\| \Gamma(q+2)}{\Gamma(q-r+1) \Gamma(p+q+1)(1-\omega)}+\frac{\|\kappa\| \Gamma(q+2) \sum_{i=1}^{n}\left|\alpha_{i}\right| \eta_{i}^{p+q}}{\Gamma(q-r+2) \Gamma(p+q+1)} \\
& \left.+\frac{|\mu| \Gamma(q+2) \mid \sum_{i=1}^{n} \eta_{i}^{q}{ }^{q}}{\Gamma(q-r+2) \Gamma(q+1)(1-\omega)}+\frac{\Gamma(q+2) \sum_{i=1}^{n}\left|\beta_{i}\right|\left|\eta_{i}\right|}{\Gamma(q-r+2)(1-\omega)}\right\} .
\end{aligned}
$$

This implies:

$$
\begin{aligned}
\|F x\|_{X} & =\max |(F x)(t)|+\max \left|\left({ }^{c} D^{r}(F x)(t)\right)\right| \\
& \leq\|x\|_{X}\{\|\kappa\| \Theta+\mathrm{Y}\}<\|x\|_{X} .
\end{aligned}
$$

This is a contradiction, because our supposition that one is an eigenvalue of the operator $\|F x\|_{X}$ is the wrong assumption. Hence, one is not an eigenvalue of $\|F x\|_{X}$. The operator $\|T x\|_{X}$ is uniformly bounded and equicontinuous as in Theorem 1. 
Now, we prove $\frac{\|T x-F x\|_{X}}{\|x\|_{X}} \rightarrow 0$ as $\|x\|_{X} \rightarrow \infty$, then:

$$
\begin{aligned}
& \|T x-F x\| \leq \frac{1}{\Gamma(q+p)} \int_{0}^{t}(t-s)^{q+p-1}\left|f\left(s, x(s){ }^{c} D^{r} x(s)\right)-\kappa(s)\left(x(s)+{ }^{c} D^{r} x(s)\right)\right| d s \\
& +\frac{1}{|1-\omega|}\left|T_{2}(f)-T_{2}\left(\kappa\left(x+{ }^{c} D^{r} x\right)\right)\right| \\
& \leq \frac{1}{\Gamma(q+p)} \int_{0}^{t}(t-s)^{q+p-1}\left|\frac{f\left(s, x(s){ }^{c} D^{r} x(s)\right)}{x(s)+{ }^{c} D^{r} x(s)}-\kappa(s)\right|\left(|x(s)|+\left|{ }^{c} D^{r} x(s)\right| d s\right. \\
& +\frac{1}{|1-\omega|}\left[\frac{1}{\Gamma(p+q)} \int_{0}^{1}(1-s)^{q+p-1}\left|\frac{f\left(s, x(s){ }^{c} D^{r} x(s)\right)}{x(s)+{ }^{c} D^{r} x(s)}-\kappa(s)\right|\left(|x(s)|+\left|{ }^{c} D^{r} x(s)\right|\right) d s\right. \\
& \left.+\sum_{i=1}^{n} \frac{\left|\alpha_{i}\right|}{\Gamma(p+q)} \int_{0}^{\eta_{i}}\left(\eta_{i}-s\right)^{q+p-1}\left|\frac{f\left(s, x(s){ }^{c} D^{r} x(s)\right)}{x(s)+{ }^{c} D^{r} x(s)}-\kappa(s)\right|\left(|x(s)|+\left|{ }^{c} D^{r} x(s)\right|\right) d s\right] \\
& \leq\left\{\frac{1}{\Gamma(p+q+1)}+\frac{1}{\Gamma(p+q+1)(1-\omega)}+\frac{\sum_{i=1}^{n}\left|\alpha_{i}\right| \eta_{i} p+q}{\Gamma(p+q+1)|1-\omega|}\right\}\left|\frac{f\left(t, x(t){ }^{c} D^{r} x(t)\right)}{x(t)+{ }^{c} D^{r} x(t)}-\kappa(t)\right|\|x\|_{X}
\end{aligned}
$$

and similarly,

$$
\begin{aligned}
& \left\|{ }^{c} D^{r} T x-{ }^{c} D^{r} F x\right\| \leq\left\{\frac{1}{\Gamma(p+q-r+1)}+\frac{\Gamma(q+2)}{\Gamma(q-r+2) \Gamma(p+q+1)|1-\omega|}\right. \\
& \left.+\frac{\Gamma(q+2) \sum_{i=1}^{n}\left|\alpha_{i}\right| \eta_{i} p+q}{\Gamma(q-r+2) \Gamma(p+q+1)|1-\omega|}\right\}\left|\frac{f\left(t, x(t) D^{r} x(t)\right)}{x(t)+{ }^{c} D^{r} x(t)}-\kappa(t)\right|\|x\|_{X}
\end{aligned}
$$

Therefore,

$$
\begin{aligned}
& \frac{\|T x-F x\|_{X}}{\|x\|_{X}}=\frac{\|(T x)(t)-(F x)(t)\|}{\|x\|_{X}}+\frac{\left\|{ }^{c} D^{r}(T x)(t)-{ }^{c} D^{r}(F x)(t)\right\|}{\|x\|_{X}} \\
& \leq\left\{\frac{1}{\Gamma(p+q+1)}+\frac{1+\sum_{i=1}^{n}\left|\alpha_{i}\right| \eta_{i}^{p+q}}{(1-\omega) \Gamma(p+q+1)}+\frac{1}{\Gamma(p+q-r+1)}+\frac{\Gamma(q+2)\left(1+\sum_{i=1}^{n}\left|\alpha_{i}\right| \eta_{i}^{p+q}\right)}{(1-\omega) \Gamma(q-r+2) \Gamma(p+q+1)}\right\} \\
& \times\left|\frac{f\left(t, x(t){ }^{c} D^{r} x(t)\right)}{x(t)+{ }^{c} D^{r} x(t)}-\kappa(t)\right|
\end{aligned}
$$

By assumption G4,

$$
\lim _{\|x\| \rightarrow \infty} \frac{\|T x-F x\|_{X}}{\|x\|_{X}}=0
$$

Then, Krasnoselskii-Zabreiko's fixed point theorem leads to the existence of a solution for the boundary value problem Equations (1) and (2).

We present now the Banach contraction principle to prove the uniqueness of the solution for problem Equations (1) and (2).

Theorem 3. Assume that $G_{1}$ and $G_{5}$ hold. The boundary value problem Equations (1) and (2) have a unique solution if $\sigma<1$ where:

$$
\sigma=L \Theta+Y
$$

and $\Theta$ and $\mathrm{Y}$ are defined as in Equations (7) and (8), respectively. 
Proof. Using the assumption $G_{5}$, we have:

$$
\begin{aligned}
& |(T x)(t)-(T \hat{x})(t)| \leq \frac{1}{\Gamma(q+p)} \int_{0}^{t}(t-s)^{q+p-1}\left|f\left(s, x(s),{ }^{c} D^{r} x(s)\right)-f\left(s, \hat{x}(s),{ }^{c} D^{r} \hat{x}(s)\right)\right| d s \\
& +\frac{|\mu|}{\Gamma(q)} \int_{0}^{t}(t-s)^{q-1}|x(s)-\hat{x}(s)| d s \\
& +\frac{1}{|1-\omega|}\left(\left|T_{1}(x)-T_{1}(\hat{x})\right|+\left|T_{2}\left(f\left(s, x(s),{ }^{c} D^{r} x(s)\right)\right)-T_{2}\left(f\left(s, \hat{x}(s),{ }^{c} D^{r} \hat{x}(s)\right)\right)\right|\right) \\
& \leq L\|x-\hat{x} \mid\|_{X}\left\{\frac{t^{p+q}}{\Gamma(p+q+1)}+\frac{1}{|1-\omega| \Gamma(p+q+1)}+\frac{\sum_{i=1}^{n}\left|\alpha_{i}\right| \eta_{i}^{p+q}}{|1-\omega| \Gamma(p+q+1)}\right\} \\
& +\|x-\hat{x}\|_{X}\left\{\frac{|\mu| t^{q}}{\Gamma(q+1)}+\frac{t^{q+1}|\mu|}{|1-\omega| \Gamma(q+1)}+\frac{t^{q+1}|\mu|}{|1-\omega| \Gamma(q+1)} \sum_{i=1}^{n}\left|\alpha_{i}\right| \eta_{i}^{q}+\frac{1}{|1-\omega|} \sum_{i=1}^{n}\left|\beta_{i}\right|\left|\eta_{i}\right|\right\} \\
& \leq\left(L \Theta_{p, 0}+\mathrm{Y}_{0}\right)\|x-\hat{x}\|_{X}
\end{aligned}
$$

likewise,

$$
\left|{ }^{c} D^{r}(T x)(t)-{ }^{c} D^{r}(T \hat{x})(t)\right| \leq\left(L \Theta_{p, r}+Y_{r}\right)\|x-\hat{x}\|_{X}
$$

Hence,

$$
\begin{aligned}
\|T x-T \hat{x}\|_{X} & =\max _{t \in[0,1]}|(T x)(t)-(T \hat{x})(t)|+\max _{t \in[0,1]}\left|\left({ }^{c} D^{r}(T x)(t)\right)-\left({ }^{c} D^{r}(T \hat{x})(t)\right)\right| \\
& \leq\left(L \Theta_{p, 0}+\mathrm{Y}_{0}\right)\|x-\hat{x}\|_{X}+\left(L \Theta_{p, r}+Y_{r}\right)\|x-\hat{x}\|_{X} \\
& \leq(L \Theta+\mathrm{Y})\|x-\hat{x}\|_{X} \leq \sigma\|x-\hat{x}\|_{X}
\end{aligned}
$$

Since $\sigma<1$, then the operator $T x$ is a contraction. Therefore, from the contraction mapping principle, the boundary value problem Equations (1) and (2) have a unique solution on $[0,1]$.

\section{Example}

Example 1. Consider the following boundary value problem:

$$
\left\{\begin{array}{l}
{ }^{c} D^{\frac{9}{8}}\left({ }^{c} D^{\frac{5}{8}}+\frac{1}{10}\right) x(t)=f\left(t, x(t), D^{\frac{3}{8}} x(t)\right), \quad 0<t<1 \\
x(0)=0, \quad{ }^{c} D^{\frac{5}{8}} x(0)=0, \\
x(1)=\frac{1}{4} x\left(\frac{1}{3}\right)+\frac{1}{2} x\left(\frac{1}{9}\right)+\frac{1}{5} \int_{0}^{1 / 3} x(s) d s+\frac{2}{5} \int_{0}^{1 / 9} x(s) d s .
\end{array}\right.
$$

We choose $p=9 / 8, q=5 / 8, r=3 / 8, \alpha_{i}=\frac{i}{4}, \beta_{i}=\frac{i}{7}, \eta_{i}=\frac{1}{3^{2}},(i=1,2)$, and $\mu=1 / 4$. Define the continuous function by:

$$
f(t, x, y)=\frac{e^{t} \sin (\pi t)}{(2+t)^{3}}+\frac{t \cos ^{2} \pi t}{(3-t)^{4}}(x+y)
$$

Observe that the function $f$ is continuous and $f(t, 0,0)=\frac{e^{t} \sin (\pi t)}{(2+t)^{3}} \neq 0$ on $(0,1)$, which means that the assumptions $G_{1}$ and $G_{3}$ hold. Now, we have:

$$
\frac{f(t, x, y)}{x+y}=\frac{e^{t} \sin (\pi t)}{(2+t)^{3}(x+y)}+\frac{t \cos ^{2} \pi t}{(3-t)^{4}}
$$

which implies that:

$$
\lim _{\|x\|_{X} \rightarrow \infty} \frac{f(t, x, y)}{x+y}=\frac{t \cos ^{2} \pi t}{(3-t)^{4}}=\kappa(t) \text { and }\|\kappa\|=\frac{1}{16} .
$$


Thus, we can calculate $\|\kappa\| \Theta+Y \sim \frac{1}{16}(3.01664)+0.75232=0.94086<1$. Therefore, by Theorem 2, the boundary value problem Equations (1) and (2) have a solution in $[0,1]$.

Furthermore, it is clear that the function $f$ satisfies the assumption $G_{5}$ with $L=1 / 16$ and $\sigma=L \Theta+Y \sim$ $0.94086<1$. Then, by Theorem 3, the boundary value problem Equations (1) and (2) have a unique solution in $[0,1]$.

\section{Conclusions}

The existence and uniqueness of the solution for the fractional nonlinear Langevin equation of two different fractional orders under the boundary conditions containing multi-point and multi-strip were studied. We found an equivalence of the problem by using the tools of fractional calculus and fixed point theorems. To examine our problem, we employed Krasnoselskii-Zabreiko, Schauder, and Banach contraction fixed point theorems. Our method was simple and appropriate for a diversity of real-world problems by choosing different forms of the function $f$ in the Langevin equation. For instance, if $\left.f=-\gamma(x(t))^{c} D^{r} x(t)\right)+\eta(x(t)) \xi(t)+F(t, x(t))$ where $\gamma(x(t))$ is the position-dependent phenomenological fluid friction coefficient, $F(z, t)$ is the external force field, $\eta(x(t))$ is the intensity of the stochastic force, and $\xi(t)$ is a zero-mean Gaussian white noise term, then the model describes the fractional Markovian set of stochastic differential equations [42].

Author Contributions: Conceptualization, methodology, formal analysis, A.S.; investigation, writing-original draft preparation, B.A. All authors have read and agreed to the published version of the manuscript.

Funding: This project was funded by the Deanship of Scientific Research (DSR), King Abdulaziz University, Jeddah. The authors, therefore, acknowledge with thanks DSR technical and financial support.

Conflicts of Interest: The authors declare no conflict of interest.

\section{References}

1. Coffey, W.T.; Kalmykov, Y.P.; Waldron, J.T. The Langevin Equation: With Applications to Stochastic Problems in Physics, Chemistry and Electrical Engineering; World Scientific: Singapore, 2004.

2. Lim, S.C.; Teo, L.P. The fractional oscillator process with two indices. J. Phys. A Math. Theor. 2009, 42, 065208. [CrossRef]

3. Lim, S.C.; Li, M.; Teo, L.P. Langevin equation with two fractional orders. Phys. Lett. 2008, 372, 6309-6320. [CrossRef]

4. Eab, C.H.; Lim, S.C. Fractional Langevin equation of distributed order. arXiv 2010, arXiv:1010.3327.

5. Sandev, T.; Tomovski, Z. Fractional Equations and Models: Theory and Applications; Springer Nature: Geneva, Switzerland, 2019.

6. West, B.J.; Bologna, M.; Grigolini, P. Physics of Fractal Operators; Springer: New York, NY, USA, 2003.

7. Kobelev, V.; Romanov, E. Fractional Langevin equation to describe anamalous diffusion Prog. Theor. Phys. Suppl. 2000, 139, 470-476. [CrossRef]

8. Sandev, T.; Metzler, R.; Tomovski, Z. Correlation functions for the fractional generalized Langevin equation in the presence of internal and external noise. J. Math. Phys. 2014, 55, 023301. [CrossRef]

9. Sandev, T.; Metzler, R.; Tomovski, Z. Velocity and displacement correlation functions for fractional generalized Langevin equations. Fract. Calc. Appl. Anal. 2012, 15, 426. [CrossRef]

10. West, B.J. Fractal physiology and the fractional calculus: A perspective. Front. Physiol. 2010, 1, 12. [CrossRef]

11. Kolmogorov, A.N. Wienersche Spiralen und einige andere interessante Kurvenim Hilbertschen Raum. Dokl. Acad. Sci. USSR 1940, 26, 115.

12. Mandelbrot, B.B.; van Ness, J.W. Fractional Brownian motions, fractional noises and applications. SIAM Rev. 1968, 10, 422. [CrossRef]

13. Mainardi, F.; Pironi, P. The fractional Langevin equation:Brownian motion revisted. Extracta Math.1996, 10, 140-154.

14. Camargo, R.F.; Chiacchio, A.O.; Charnet, R.; Oliveira, E.C. Solution of the fractional Langevin equation and the Mittag-Leffler functions. J. Math. Phys. 2009, 50, 063507. [CrossRef] 
15. Vinales, A.D.; Desposito, M.A. Anomalous diffusion induced by a Mittag-Leffler correlated noise. Phys. Rev. E 2007, 75, 042102. [CrossRef] [PubMed]

16. Guo, P.; Zeng, C.; Li, C.; Chen, Y. Numerics for the fractional Langevin equation driven by the fractional Brownian motion. Fract. Calc. Appl. Anal. 2013, 16, 123-141. [CrossRef]

17. Guo, P.; Li, C.P.; Zeng, F.H. Numerical simulation of the fractional Langevin equation. Therm. Sci. 2012, 16, 357-363. [CrossRef]

18. Zhao, J.; Lu, P.; Liu, Y. Existence and Numerical Simulation of Solutions forFractional Equations Involving Two Fractional Orders withNonlocal Boundary Conditions. J. Appl. Math. 2013, 2013, 268347. [CrossRef]

19. Mahmudov, N.I. Fractional Langevin type delay equations with two fractional derivatives. Appl. Math. Lett. 2020, 103, 106215. [CrossRef]

20. Baghani, H.; Nieto, J.J. On fractional Langevin equation involving two fractional orders in different intervals. Nonlinear Anal. Model. Control. 2019, 24, 884-897. [CrossRef]

21. Zhai, C.; Li, P. Nonnegative Solutions of Initial Value Problems for Langevin Equations Involving Two Fractional Orders. Mediterr. J. Math. 2018, 15, 164. [CrossRef]

22. Baghani, $\mathrm{H}$. Existence and uniqueness of solutions to fractional Langevin equations involving two fractional orders. J. Fixed Point Theory Appl. 2018, 20, 63. [CrossRef]

23. Fazli, H.; Nieto, J.J. Fractional Langevin equation with anti-periodic boundary conditions. Chaos Solitons Fractals 2018, 114, 332-337. [CrossRef]

24. Zhai, C.; Li, P.; Li, H. Single upper-solution or lower-solution method for Langevin equations with two fractional orders. Adv. Differ. Equ. 2018, 360, 1-10. [CrossRef]

25. Baghani, O. On fractional Langevin equation involving two fractional orders. Commun. Nonlinear Sci. Numer. Simulat. 2017, 42, 675-681. [CrossRef]

26. Cetin, E.; Topa, F.S. Existence Results for Solutions of Integral Boundary Value Problems on Time Scales. Abstr. Appl. Anal. 2013, 708734, 7. [CrossRef]

27. Salem, A.; Alzahrani, F.; Alnegga, M. Coupled System of Non-linear Fractional Langevin Equations with Multi-point and Nonlocal Integral Boundary Conditions. Math. Probl. Eng. 2020, 7345658, 15.

28. Salem, A.; Alzahrani, F.; Almaghamsi, L. Fractional Langevin equation with nonlocal integral boundary condition. Mathematics 2019, 7, 402. [CrossRef]

29. Zhou, Z.; Qiao, Y. Solutions for a class of fractional Langevin equations with integral and anti-periodic boundary conditions. Bound. Value Probl. 2018, 2018, 152. [CrossRef]

30. Salem, A.; Alzahrani, F.; Alghamdi, B. Langevin equation involving two fractional orders with three-point boundary conditions. Differ. Integral Equ. 2020, 33, 163-180.

31. Salem, A.; Alghamdi, B. Multi-Point and Anti-Periodic Conditions for Generalized Langevin Equation with Two Fractional Orders. Fractal Fract. 2019, 3, 51. [CrossRef]

32. Derbazi1, C.; Hammouche, H.; Benchohra, M.; Zhou, Y. Fractional hybrid differential equations withthree-point boundary hybrid conditions. Adv. Differ. Equ. 2019, 2019, 125. [CrossRef]

33. Lv, Z.-W. Existence of Positive Solution for Fractional Differential Systems with Multi-point Boundary Value Conditions. J. Funct. Spaces Vol. 2020, 9520430, 9.

34. Sandin, T.R. The jerk. Phys. Teach. 1998, 28, 36-40. [CrossRef]

35. Schot, S.H. Jerk: The time rate of change of acceleration. Am. J. Phys. 1978, 46, 1090-1094. [CrossRef]

36. Schot, S.H. Aberrancy: Geometry of the Third Derivative. Math. Mag. 1978, 51, 259-275. [CrossRef]

37. Granas, A.; Dugundji, J. Fixed Point Theory; Springer: New York, NY, USA, 2003.

38. Kilbas, A.A.; Srivastava, H.M.; Trujillo, J.J. Theory and Applications of Fractional Differential Equations; Elsevier: Amsterdam, The Netherlands, 2006.

39. Podlubny, I. Fractional Differential Equations. In Mathematics in Science and Engineering; Academic Press: New York, NY, USA, 1999; Volume 198.

40. Su, X. Boundary value problem for a coupled system of nonlinear fractional differential equations. Appl. Math. Lett. 2009, 22, 64-69. [CrossRef] 
41. Krasnoselski, M.A.; Zabreiko, P.P. Geometrical Methods of Nonlinear Analysis; Springer: New York, NY, USA, 1984.

42. Olivares-Rivas, W.; Colmenares, P.J. The generalized Langevin equation revisited: Analytical expressions for the persistence dynamics of a viscous fluid under a time dependent external force. Phys. A 2016, 458, 76-94. [CrossRef] 